\title{
INFORMATION IN A PHOTON: RELATING ENTROPY AND MAXIMUM-LIKELIHOOD RANGE ESTIMATION USING SINGLE-PHOTON COUNTING DETECTORS
}

\author{
Dongeek Shin Ahmed Kirmani Vivek K Goyal Jeffrey H. Shapiro \\ Research Laboratory of Electronics, Massachusetts Institute of Technology
}

\begin{abstract}
Range estimation at low light-levels is accomplished using pulsed illumination of the target and time-of-flight measurement of backscattered light using single-photon detectors. Photon arrival statistics for this problem are time-inhomogeneous Poisson point processes where the rate function is determined by the illumination waveform. Given the flexibility to choose from different illumination waveforms, an important design question is - how does the range estimation performance depend on the pulse shape?

The maximum-likelihood (ML) range estimation problem is nonlinear and thus it is difficult to analytically compare the estimation performance from different illumination waveforms. In this paper, we present an information-theoretic framework for evaluating ML range estimation performance. We derive relationships between the entropy of the photon arrival observations and the Cramér-Rao lower bound (CRLB) on the range estimate by extending De Brujin's identity and isoperimetric properties for non-Gaussian distributions.
\end{abstract}

Index Terms - Low light-level range imaging, single-photon imaging, time-of-flight, differential entropy, Cramér-Rao lower bound, De Bruijn's identity.

\section{INTRODUCTION}

Light detection and ranging (LIDAR) systems allow range imaging at low light-levels [1, 2, 3] and stand-off range sensing over long distances $[4,5]$. LIDAR systems operate on the time-of-flight principle [1] - the target is illuminated with a series of identical light pulses, followed by time-resolved detection of the backscattered photons using time-correlated single-photon counting $[5,6]$.

At low light-levels, the photon arrival statistics are timeinhomogeneous Poisson point processes, where the rate function is determined by the pulse shape $[3,7]$, and the uncertainty or noise in photon-arrival measurements is attributed only to the pulse shape and pulse time-duration. Moreover, LIDAR image acquisition is point-wise or pixel-wise - it involves raster scanned illumination of one scene point at a time or imaging multiple scene points in parallel using a $2 \mathrm{D}$ sensor array. In either case, the $2 \mathrm{D}$ range image is a collection of point-wise estimates. In this setting, it is meaningful to compare point-wise range estimation accuracy achieved using different pulse shapes of the same time-duration.

The problem of range estimation from time-of-flight data in presence of additive Gaussian noise is a well studied problem [7, 8]. However, to the authors' best knowledge, detailed statistical analysis of ML range estimation using single-photon detectors is unavailable. Our work is motivated by statistical analysis introduced by Erkmen et al. [9], which approached the problem from an estimationtheoretic perspective, and the work of Guo et al. [10] which explored

This material is based upon work supported by the National Science Foundation under grant No. 1161413, a Qualcomm Innovation Fellowship, and a Samsung Scholarship.

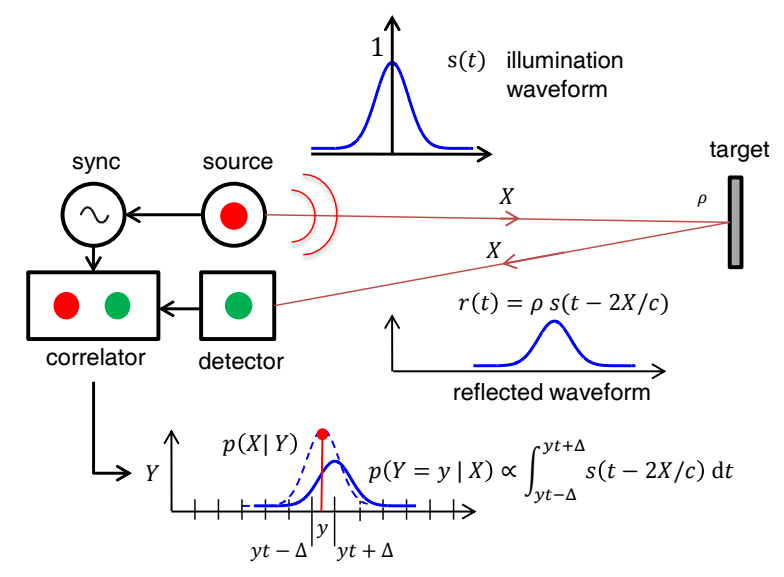

Fig. 1. Setup of the single-photon range acquisition system.

the connection between mutual information and minimum meansquared error (MMSE) in the presence of additive Gaussian noise.

Our setup is shown in Figure 1. The goal is to compute the ML estimate of target range, $X$, from $n$ photon-arrival time measurements denoted by a random vector, $Y=\left[y_{1}, \ldots, y_{n}\right]$, with i.i.d. entries. It is common to assume that the target lies within a certain range interval. Moreover, the target range, $X$, is a non-random parameter. A standard technique for ML estimation error analysis is to reformulate the ML problem as a maximum-a posteriori (MAP) problem, which is accomplished by imposing a uniform prior on $X$, i.e., $X \sim U\left[d_{\min }, d_{\max }\right]$.

Different illumination waveforms, denoted by $s(t)$, result in different ML range estimation problems. In general, the resulting ML estimation is nonlinear and it is difficult to provide analytical guarantees on range estimation performance, i.e., mean-squared error (MSE) of the ML estimator, and compare different illumination waveforms.

Our contributions are outlined as follows:

1. We analytically compute the CRLB for ML range estimation using pulse shapes described by the generalized Gaussian family of distributions representing symmetric pulses and the Gamma distribution representing skewed pulses.

2. For these pulse families, we derive two relationships - one between the CRLB and the conditional entropy, $h(Y \mid X)$, of observations $Y$ given the range parameter $X$; and the second relating the CRLB to the conditional entropy power, $N(Y \mid X)$.

From an estimation-theoretic perspective, the aforementioned results allow us to compare the range estimation performance of different pulse shapes only using the entropy of the distribution induced by the pulse shape, without requiring analytic computation of range estimation errors.

From an information-theoretic perspective, our work explores a deeper connection between information quantities - such as entropy 
- and estimation error in the context of low light-level single-photon range imaging. The derivation of our stated results required an extension of two key results in information theory - De Brujin's identity [11] and the isoperimetric equality [12] - both of which are valid only for Gaussian channels. Our work extends both of these results to a uniform source prior and channels that induce posterior distributions belonging to the generalized Gaussian family or the Gamma distribution.

\section{PROBLEM SETUP}

The intensity of the transmitted light pulse is denoted using $s(t)$. In this paper, we consider the case in which there is no ambient or background light, no detector dark counts, and the number of backscattered photons incident at the detector is much smaller than the number of transmitted pulses. Under these assumptions the reflected light is modeled as the waveform $r(t)=\rho s(t-2 x / c)$ where $x$ is the target range, $\rho$ is the target reflectivity and $c$ is the speed of light. For the simplicity of exposition, we re-normalize the time-axis to obtain $r(t)=\rho s(t-x)$. The detector measures photon time-ofarrival within a small interval $\Delta$. In practice, $\Delta$ is so small (few picoseconds) that photon arrivals are effectively continuous in time.

It is well known from the Poisson point process theory [7] that under the aforementioned conditions the photon time bin-of-arrival, $Y$, has the likelihood distribution

$$
p_{Y \mid X}(y \mid x) \propto s(y-x), \quad y>0, \quad x \in\left[d_{\min }, d_{\max }\right] .
$$

This distribution is shown in Figure 1. The constant of proportionality is equal to the total area under the pulse $s(t)$. Denote the variance of $p_{Y \mid X}(y \mid x)$ with $\sigma^{2}$. Clearly, $\sigma$ is the root mean square (RMS) time-duration of the pulse. In this, paper we only consider the case when $\sigma \ll\left(d_{\max }-d_{\min }\right)$. This assumption is typical and necessary, since we intend to achieve high range accuracy relative to the target range interval length. Because of this small $\sigma$ assumption, we ignore the boundary conditions arising while defining various probability distributions in this paper. For example, the posterior distribution then takes the form

$$
p_{X \mid Y}(x \mid y) \propto s(-x+y), \quad x \in\left[d_{\min }, d_{\max }\right] .
$$

Equation 2 indicates that the posterior distribution is proportional to time-reversed pulse shape. Thus, analysis of single photon range imaging necessitates the study of general channel models going beyond the standard Gaussian and Poisson observation channels [13]. In this paper, we undertake the study of pulse shapes that induce posterior distributions belonging to the family of generalized Gaussian and Gamma distributions. In order to compare the ML estimation performances of these pulse shapes, we henceforth consider pulse shapes with identical pulse RMS time-duration, $\sigma$.

\section{BACKGROUND}

\subsection{Maximum Likelihood Range Estimation}

The traditional ML estimator for pulsed range estimation is known as log-matched filter [7]. In the setting of single photon imaging, we use the a priori knowledge of the pulse shape to recover the target based on $n$ independent observations of single photon arrivals. Because we detect less than one mean number of backscattered photons per transmitted pulse, the traditional log-matched filter is analogous to our constrained maximum-log likelihood estimation problem:

$$
\begin{array}{cl}
\hat{x}_{\mathrm{ML}}=\underset{x}{\operatorname{argmax}} & \sum_{i=1}^{n} \log p_{Y \mid X}\left(y_{i} \mid x\right) \\
\text { s.t. } & x \in\left[d_{\min }, d_{\max }\right]
\end{array}
$$

In the absence of background light and detector dark counts, the likelihood of observing a photon arrival time outside the interval $\left[d_{\min }, d_{\max }\right]$ is negligible. Then the solution to the constrained ML problem in Equation 3, coincides with the unconstrained ML estimate,

$$
\hat{x}_{\mathrm{ML}}=\underset{x}{\operatorname{argmax}} \sum_{i=1}^{n} \log s\left(y_{i}-x\right) .
$$

It is well known that the unconstrained ML estimator defined in Equation 4 is unbiased, and is an asymptotically efficient estimator whose MSE is bounded using CRLB, which is the reciprocal of Fisher information. The CRLB for the range estimation problem is

$$
\operatorname{CRLB}(x)=-\frac{1}{n}\left(\mathbb{E}\left[\frac{d^{2}}{d x^{2}} \log p_{Y \mid X}(y \mid x)\right]\right)^{-1} .
$$

For an arbitrary likelihood $p_{Y \mid X}$, induced by a pulse shape with variance $\sigma^{2}$, Dembo et al. [12] showed that CRLB $\leq \sigma^{2} / n$ and its equality holds if and only if the likelihood is a Gaussian distribution.

\subsection{Related Information Theory}

When $n=1$, the conditional entropy over the single photon detection time variable $Y$ given prior variable $X$ is defined as

$$
h(Y \mid X)=-\int_{-\infty}^{+\infty} \int_{d_{\min }}^{d_{\max }} p_{X, Y}(x, y) \log p_{Y \mid X}(y \mid x) d x d y .
$$

When $X$ is uniformly distributed and $\sigma$ is small relative to length of the interval prior, the conditional entropy expression simplifies as,

$h(Y \mid X)=-\int_{-\infty}^{+\infty}\left(\frac{s(y)}{\int_{-\infty}^{+\infty} s\left(y^{\prime}\right) d y^{\prime}}\right) \log \left(\frac{s(y)}{\int_{-\infty}^{+\infty} s\left(y^{\prime}\right) d y^{\prime}}\right) d y$.

We discuss two relationships that relate entropy with the Fisher information: De Brujin's identity and the isoperimetric inequality. These inequalities will be used as part of our analysis in Section 4.

De Brujin's identity [11] relates entropy $h(Z)$ with the Fisher information $J(Z)$, where $Z$ is the output variable of a Gaussian channel with arbitrary prior variable, as

$$
\frac{d}{d v} h(Z)=\frac{1}{2} J(Z), \quad \text { where } v \text { is variance of } Z \text {. }
$$

Another relationship between entropy and Fisher information is the isoperimetric inequality [12]. The isoperimetric inequality states that

$$
\frac{1}{n} N(Z) J(Z) \geq 1
$$

for any size $n$ random vector $Z$ and its entropy power $N(Z)$ defined as

$$
N(Z)=\frac{1}{2 \pi e} \exp \left\{\frac{2}{n} h(Z)\right\} .
$$

The equality in the isoperimetric inequality holds if and only if the likelihood is a Gaussian distribution, which is the maximum entropy distribution among all distributions with fixed variance.

For Gaussian channels, the relationship between the entropy and the CRLB is clearly understood by De Brujin's identity and the isoperimetric equality. However, similar relationships have not been studied for non-Gaussian pulse shapes that are frequently used in low light-level range estimation. In order to evaluate the performance of ML estimators using expressions of conditional entropy, we derive extensions to the De Brujin's and isoperimetric identities for generalized Gaussian and Gamma shaped pulses assuming a uniform prior on the range. 


\section{RELATIONSHIP BETWEEN ENTROPY AND CRAMÉR-RAO BOUND}

\subsection{Generalized Gaussian Channels}

If our pulse, $s(t)$, belongs to the generalized Gaussian family, the likelihood function from a single-photon observation is,

$$
p_{Y \mid X}(y \mid x)=\frac{1}{2 a \Gamma(1+1 / p)} \exp \left\{-\left(\frac{|y-x|}{a}\right)^{p}\right\}
$$

where $a>0$ is the scale parameter, $p \geq 1$ is the order parameter, and $\Gamma(x)=\int_{0}^{\infty} t^{x-1} e^{-t} d t$ is the gamma function. Parameter $a$ controls the pulse width, and $p$ controls the concavity and pulse shape. The generalized Gaussian distribution (see Figure 2) includes pulses frequently used in practical range imaging systems such as the Gaussian pulse $(p=2)$, and near-uniform pulse (large $p$ ).

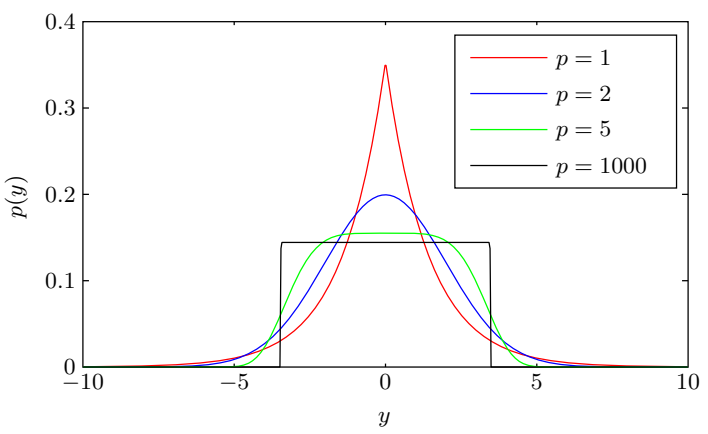

Fig. 2. Generalized Gaussian distributions for $p=1,2,5,1000$ and fixed variance. Higher $p$ reduces concavity and tailing behavior in the distribution.

The conditional variance is $\operatorname{Var}(Y \mid X=x)=a^{2} \Gamma(3 / p) / \Gamma(1 / p)$, denoted by $\sigma^{2}$. The unconstrained ML estimate of the target range using a generalized Gaussian pulse is obtained by solving an optimization problem which is strictly convex if $p>1$. The CRLB for ML range estimation using generalized Gaussian pulse shapes with $1<p<\infty$ is computed to be the following expression:

$$
\mathrm{CRLB}=\left(\frac{\Gamma(1 / p)^{2}}{p(p-1) \Gamma(3 / p) \Gamma(1-1 / p)}\right) \frac{\sigma^{2}}{n} .
$$

We also have the conditional entropy [13] as

$$
h(Y \mid X)=n\left(\frac{1}{p}+\log \left(2 \Gamma\left(1+\frac{1}{p}\right) \sqrt{\frac{\Gamma(1 / p)}{\Gamma(3 / p)}} \sigma\right)\right) .
$$

In the setting considered in the paper, there are no photon arrivals due to background or detector dark count. The only source of uncertainty or noise in photon-arrival measurements is attributed only to the pulse shape and pulse time-duration. Therefore, it is natural to define $\mathrm{SNR}=1 / \sigma^{2}$. We note that both $h(Y \mid X)$ and CRLB are non-increasing and strictly convex with respect to SNR.

We then derive an identity that relates the derivative of the entropy to CRLB,

$$
\begin{aligned}
& \frac{d}{d \mathrm{SNR}} h(Y \mid X)=\xi_{1} \mathrm{CRLB}, \\
& \text { where } \xi_{1}=-\frac{n^{2} p(p-1) \Gamma(3 / p) \Gamma(1-1 / p)}{2 \Gamma(1 / p)^{2}} .
\end{aligned}
$$

Equation 14 extends De Brujin's identity to generalized Gaussian channels. However, our result is only valid for uniform source prior whereas De Brujin's identity holds for arbitrary source distributions but only for the Gaussian channel. Setting $n=1$ and $p=2$ reduces Equation 14 to the classical De Brujin's identity.

Also, Figure 3 shows that the non-monotone trends in $N(Y \mid X)$ and CRLB with respect to $p$ agree. It confirms the maximum entropy
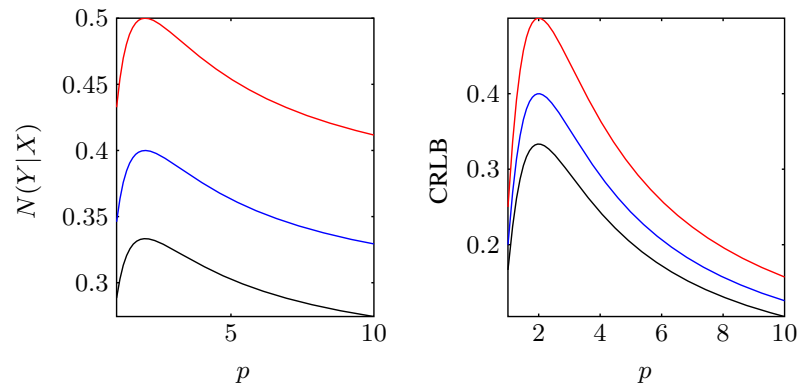

Fig. 3. Plot of $N(Y \mid X)$ and CRLB w.r.t $p$ for generalized Gaussian pulses. The red, blue, and black lines have SNR values 2, 2.5, and 3, respectively.

and maximum Cramér-Rao bound principles of the Gaussian variable by showing $\operatorname{argmax}_{p>1} N(Y \mid X)=\operatorname{argmax}_{p>1}$ CRLB $=2$. For generalized Gaussian pulses, the entropy power is related to the CRLB as

$$
\begin{aligned}
& N(Y \mid X)=\xi_{2} \text { CRLB }, \\
& \text { where } \xi_{2}=n \frac{2 p(p-1) \Gamma(1+1 / p)^{2} \Gamma(1-1 / p)}{\pi e \Gamma(1 / p)} \exp \left\{\frac{2}{p}\right\} .
\end{aligned}
$$

Equation 15 extends the classical isoperimetric equality to the generalized Gaussian distribution. Note that $p=2$ implies $\xi_{2}=n$, giving the isoperimetric equality of the Gaussian distribution.

\subsection{Gamma Channels}

We now consider pulse shapes with Gamma distribution (Figure 4).

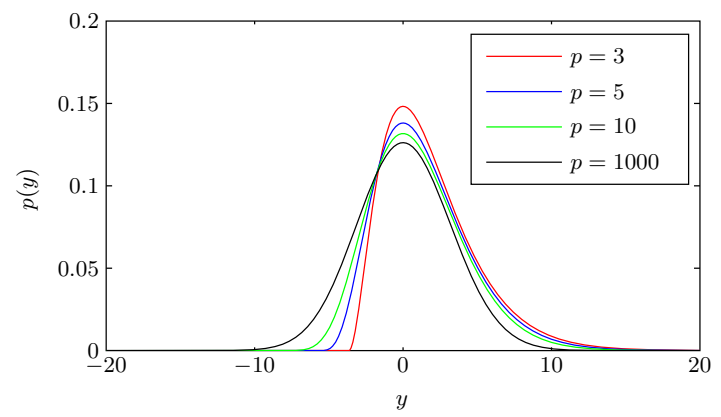

Fig. 4. Mode shifted Gamma distributions for $p=3,5,10,1000$ and fixed variance. Lower $p$ implies higher skew of the distribution.

The likelihood from a single observation can be written as

$$
p_{Y \mid X}(y \mid x)=\frac{1}{\Gamma(p) \lambda^{p}}(y-x+k)^{p-1} \exp \left\{-\frac{y-x+k}{\lambda}\right\},
$$

where $\lambda>0$ is the scale parameter, $p>1$ is the parameter that controls the distribution skew, and $k=(p-1) \lambda$ is the mode of Gamma distribution so that $y-x+k>0$. Because Gamma distributions are log-concave, the ML estimate is obtained by solving a convex optimization problem. Assuming $p>2$ for regularity conditions, the CRLB that bounds this ML estimate is

$$
\mathrm{CRLB}=\left(\frac{\Gamma(p)}{p(p-1) \Gamma(p-2)}\right) \frac{\sigma^{2}}{n},
$$

and the conditional entropy is

$$
h(Y \mid X)=n\left(\log \sigma+\log \frac{\Gamma(p)}{\sqrt{p}}+p+(1-p) \psi(p)\right),
$$

where $\psi(x)=d \log \Gamma(x) / d x$ is the digamma function. We see that both $h(Y \mid X)$ and CRLB are non-increasing and strictly convex with respect to $\mathrm{SNR}=1 / \sigma^{2}$. 

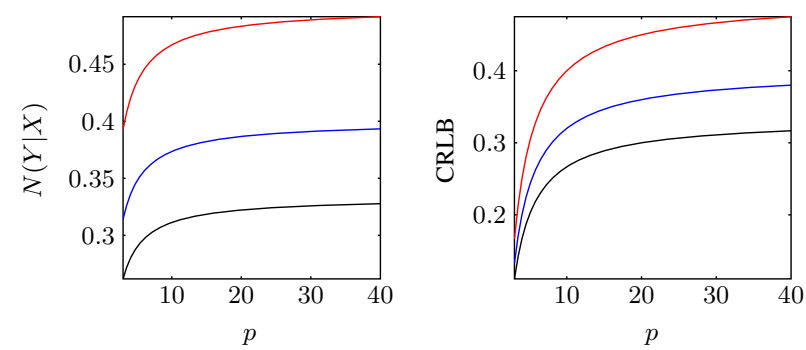

Fig. 5. Plot of $N(Y \mid X)$ and CRLB w.r.t $p$ for Gamma pulses. The red, blue, and black lines have SNR values 2, 2.5, and 3, respectively.

We can then observe a derivative relationship that relates the entropy and CRLB as

$$
\begin{aligned}
& \frac{d}{d \mathrm{SNR}} h(Y \mid X)=\xi_{3} \mathrm{CRLB}, \\
& \text { where } \xi_{3}=-\frac{n^{2} p(p-1) \Gamma(p-2)}{2 \Gamma(p)} .
\end{aligned}
$$

As $p \rightarrow \infty$ in Equation 19 and the Gamma distribution becomes normal, one can approximate Gamma functions using Stirling's formula and confirm De Brujin's identity.

Figure 5 plots the concavity trends in $N(Y \mid X)$ and the CRLB with respect to $p$ and shows that they agree. We also observe a direct relationship between the entropy power and the CRLB.

$$
\begin{aligned}
& N(Y \mid X)=\xi_{4} \text { CRLB, } \\
& \text { where } \xi_{4}=n \frac{(p-1) \Gamma(p-2) \Gamma(p)}{2 \pi e} \exp \{2 p+2(1-p) \psi(p)\} .
\end{aligned}
$$

Similarly, notice that $p \rightarrow \infty$ implies $\xi_{4} \rightarrow n$, and reduces Equation 20 to isoperimetric equality for the Gaussian distribution.

\section{SIMULATIONS AND DISCUSSION}

Figure 6 compares the CRLB (dotted lines) computed using differential entropy as defined in Equation 7, with Monte Carlo simulations of the MSE (solid lines) of the constrained ML range estimator with $n=5$. The simulations are carried for both generalized Gaussian shaped pulses of varying concavity (top), and for the Gamma distribution shaped pulses with varying skew (bottom).

The simulation results confirm that Gaussian pulse shape ( $p=$ 2 ) has the highest estimation error within the generalized Gaussian family - both in terms of simulated MSE and CRLB. However, for Gaussian pulses, the CRLB is tight. When the pulse order, $p$, is either increased or decreased from $p=2$, the MSE decreases but the discrepancy between MSE and CRLB increases. This non-monotonic trend is consistent with our information-theoretic interpretation as shown in Figure 3, which demonstrates that Gaussian pulses have the highest entropy power for the family of distributions with fixed variance [12]. The best range estimation performance within the generalized Gaussian family comes from the pulse shape approaching a rectangular pulse $(p \rightarrow \infty)$, which has a sharp rising edge. For Gamma distributions, the simulations corroborate the decrease in MSE and also the decrease in discrepancy between CRLB and MSE with increasing skewness.

The aforementioned findings are not entirely surprising - increasing skew results in sharper rising pulse edges which implies an increase in the frequency bandwidth of the pulse. It is a well known result in classical range imaging using matched filtering in presence of additive Gaussian noise, that for fixed RMS pulse duration, estimation error decreases with increasing pulse bandwidth [8]. However, this result was not explored in the context of single-photon
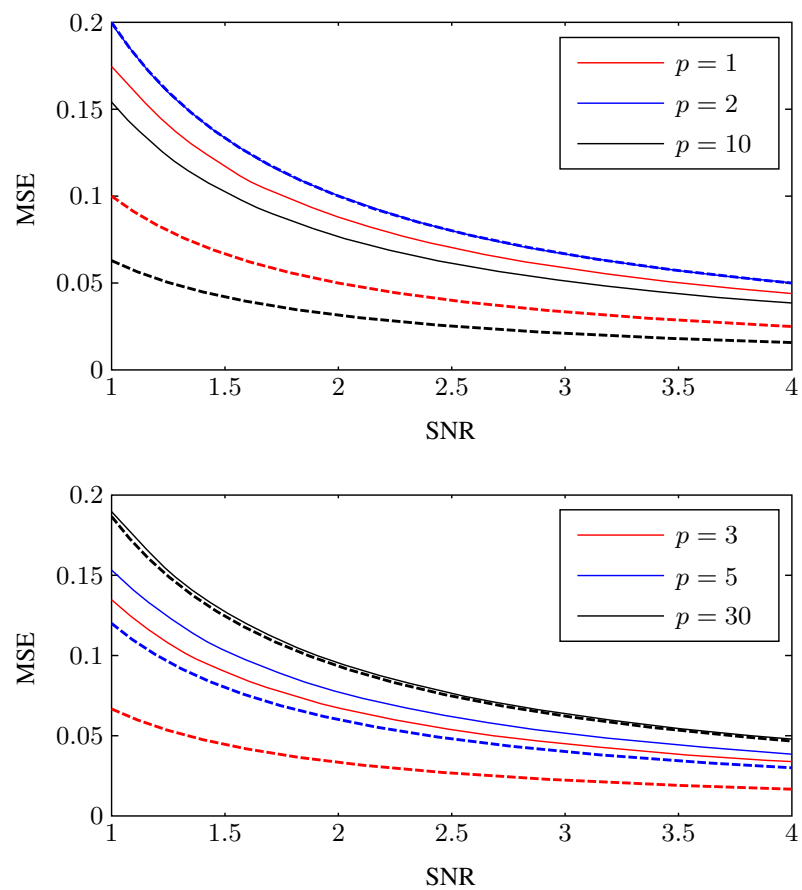

Fig. 6. Plot of MSE of the constrained ML estimates (solid lines) and CRLB computed from entropy (dotted lines) when using generalized Gaussian (top) and Gamma (bottom) shaped pulses. Note that SNR $=1 / \sigma^{2}$.

detection. Our work paves the way for principled estimation and information theoretic analysis of single-photon range imaging.

\section{CONCLUSION AND FUTURE WORK}

Low power 3D sensing is critical for a number of practical applications [14]. Moreover, low light-level LIDAR systems allow depth sensing at extremely low optical powers. As LIDAR systems are integrated into commercial solutions, it is important to understand their limitations and analyze their performance for varying design choices such as different pulse shapes.

We analyzed the problem of low light-level range estimation from single photon time-of-arrival measurements using an estimation-theoretic and information-theoretic approach. We contrast the two approaches and derive key relationships between CRLB of the ML range estimate and differential entropy of the likelihood function induced by the pulse shape assuming a uniform source distribution. Strong results that relate entropy and CRLB exist only for the Gaussian channel $[10,11]$. However, we show that the single photon range imaging setting introduces non-Gaussian channel models for which estimation-theoretic analysis is analytically intractable. By considering the example of generalized Gaussian and Gamma distributions, we show that it is possible to exploit the information quantities to analyze estimation error. We confirm that Gaussian pulses have the highest estimation error - a result that is consistent with the maximum Cramér-Rao bound principle.

Our future work comprises incorporating background noise and detector dark counts in our analysis framework. With these nonidealities, the CRLB and MSE analysis becomes more difficult analytically, and therefore use of an information-theoretic approach may facilitate simpler analysis. We also intend to consider the use of sparsity promoting priors during range estimation which would require us to go beyond point-wise analysis. 


\section{REFERENCES}

[1] B. Schwarz, "Mapping the world in 3d," Nat. Photonics, vol. 4, pp. 429-430, 2010.

[2] S. Pellegrini, G. S. Buller, J. M. Smith, A. M. Wallace, and S. Cova, "Laser-based distance measurement using picosecond resolution time-correlated single-photon counting," Measurement Science and Technology, vol. 11, no. 6, pp. 712, 2000.

[3] A. Kirmani, D. Venkatraman, A. Colaço, F. N. C. Wong, and V. K. Goyal, "High photon efficiency computational laser range imaging using spatio-temporal statistical regularization," in Lasers and Electro-Optics (CLEO), 2013 Conference on, 2013.

[4] P. J. Besl, "Active, optical range imaging sensors," Machine vision and applications, vol. 1, no. 2, pp. 127-152, 1988.

[5] A. McCarthy, R. J. Collins, N. J. Krichel, V. Fernández, A. M. Wallace, and G. S. Buller, "Long-range time-of-flight scanning sensor based on high-speed time-correlated single-photon counting," Applied Optics, vol. 48, no. 32, pp. 6241-6251, 2009.

[6] A. Colaço, A. Kirmani, G. A. Howland, J. C. Howell, and V. K. Goyal, "Compressive depth map acquisition using a single photon-counting detector: Parametric signal processing meets sparsity," in IEEE Conf. Computer Vision and Pattern Recognition (CVPR), 2012, pp. 96-102.

[7] D. L. Snyder, Random Point Processes, Wiley, New York, 1975.

[8] J. H. Shapiro, R. W. Reinhold, and D. Park, "Performance analyses for peak-detecting laser radars," in Proc. SPIE, 1986, vol. 663, pp. 38-56.

[9] B. I. Erkmen and B. Moision, "Maximum likelihood time-ofarrival estimation of optical pulses via photon-counting photodetectors," in Proc. IEEE Int. Symp. Information Theory, 2009, pp. 1909-1913.

[10] D. Guo, S. Shamai, and S. Verdú, "Mutual information and minimum mean-square error in gaussian channels," IEEE Trans. Information Theory, vol. 51, no. 4, pp. 1261-1282, 2005.

[11] A. J. Stam, "Some inequalities satisfied by the quantities of information of Fisher and Shannon," Information and Control, vol. 2, no. 2, pp. 101-112, 1959.

[12] A. Dembo, T. M. Cover, and J. A. Thomas, "Information theoretic inequalities," IEEE Trans. Information Theory, vol. 37, no. 6, pp. 1501-1518, 1991.

[13] T. M. Cover and J. A. Thomas, Elements of Information Theory, Wiley-Interscience, 2006.

[14] A. Kirmani, A. Colaço, F. Wong, and V. Goyal, "Exploiting sparsity in time-of-flight range acquisition using a single timeresolved sensor," Optics Express, vol. 19, no. 22, pp. 2148521507, 2011. 